\title{
A tailored approach for the treatment of indirect inguinal hernia in adults—an old problem revisited
}

\author{
Martin Hübner • Markus Schäfer • Hicham Raiss • \\ Nicolas Demartines $\cdot$ Henri Vuilleumier
}

Received: 11 January 2010 / Accepted: 25 February 2010 / Published online: 3 July 2010

(C) Springer-Verlag 2010

\begin{abstract}
Purpose A patent processus vaginalis peritonei (PPV) presents typically as an indirect hernia with an intact inguinal canal floor during childhood. Little is known however about PPV in adults and its best treatment.

Methods A cohort study included all consecutive patients admitted for ambulatory open hernia repair. In patients with a PPV, demographics, hernia characteristics, and outcome were prospectively assessed. Annulorrhaphy was the treatment of choice in patients with an internal inguinal ring diameter of $<30 \mathrm{~mm}$.

Results Between 1998 and 2006, 92 PPVs (two bilateral) were diagnosed in 676 open hernia repairs (incidence of $14 \%)$. Eighty nine of the 90 patients were males, the median age was 34 years (range: 17-85). A PPV was rightsided in $67 \%$ and partially obliterated in $66 \%$. Forty-one patients had an annulorrhaphy and 51 patients had a tension-free mesh repair. The median operation time was significantly shorter in the annulorrhaphy group (38 vs. 48 min, $P<.0001)$. In a median follow-up period of 56 months (27-128), both groups did not differ concerning recurrence (1/41 vs. $2 / 51)$, chronic pain (3/41 vs. $4 / 51)$, and hypoesthesia (5/41 vs. $9 / 51)$. There was however a clear trend to less neuropathic symptoms in favor of annulorrhaphy ( $0 / 41$ vs. $5 / 51, P<0.066)$.

Conclusions PPV occurs in $14 \%$ of adults undergoing hernia repair. In selected patients, annulorrhaphy takes less time and is associated with equally low recurrence but less potential for neuropathic symptoms.
\end{abstract}

M. Hübner $\cdot$ M. Schäfer $\cdot H$. Raiss $\cdot$ N. Demartines ·

H. Vuilleumier $(\triangle)$

Department of Visceral Surgery, University Hospital CHUV,

Rue du Bugnon 46,

1011 Lausanne, Switzerland

e-mail: henri.vuilleumier@chuv.ch
Keywords Inguinal hernia $\cdot$ Patent processus vaginalis · Adult · Obliteration $\cdot$ Congenital $\cdot$ Annulorrhaphy

\begin{abstract}
Abbreviations
PPV Patent processus vaginalis peritonei
\end{abstract}

\section{Introduction}

Inguinal hernia represents one of the commonest surgical pathologies worldwide. Patients of all ages, ranging from neonates to octogenarians, are affected, and a significant amount of them need to be operated [1]. In contrast to many other surgical diseases, investigations of the etiology of inguinal hernia formation have attracted much less interest than the technical aspects of surgical treatment. The precise anatomy and physiology of the groin with its impact on hernia formation remained largely unclear until Fruchaud's concept of the myopectineal orifice has become widely accepted $[2,3]$. Despite some important refinements of this concept have been made during recent years, the issue of a patent processus vaginalis (PPV) is still under debate [4-6].

The majority of groin hernias in infants and children are indirect hernias that are related to a patent processus vaginalis [7-9]. Its treatment is simple and includes solely the removal of the hernia sac, and rarely, narrowing of the internal ring [7, 10]. In contrast, the overall incidence of indirect inguinal hernia in young adults is low; however, it is increasing with age [11]. And of note, middle-aged and older patients generally present with larger indirect inguinal hernia than young adults [11]. It must be therefore strongly assumed that other factors than a patent or reopened processus vaginalis play an important role. In fact, in case of large indirect hernia, an acquired defect of the trans- 
versalis fascia is always present. The development of such fascial weakening is promoted by several risk factors, e.g., smoking, increased intra-abdominal pressure, and disturbances of the collagen metabolism [1]. Direct inguinal hernias are more often bilateral, and its occurrence is closely related to age and the aforementioned risk factors [1].

Traditionally, inguinal hernia repair by using a suture or mesh technique aimed to reconstruct and reinforce the inguinal floor, in particular the transversalis fascia $[11,12]$. However, small indirect inguinal hernia in younger patients with a limited enlargement of the inner ring and an intact transversalis fascia could eventually be repaired by reconstruction of the inner ring. This limited approach was proposed by Nyhus during the 1960s [13, 14]. Unfortunately, these early attempts revealed a high failure rate; hence, they were rapidly abandoned $[12,15]$.

The true incidence of a PPV remains largely unknown. Since the concept of simple reconstruction of the inner ring remains appealing, the issue of a pre-existing PPV must be better elucidated and clear selection criteria would be mandatory in order to achieve a better success rate of a limited surgical approach.

The current study aimed to determine the prevalence of a PPV in adults undergoing surgery for symptomatic inguinal hernia. In addition, the outcome of a tailored approach for repair of indirect inguinal hernia due to PPV was assessed.

\section{Patients and Methods}

\section{Patients}

Data of all patients undergoing elective ambulatory repair for primary inguinal hernia in our department from April 1998 to December 2006 were prospectively collected in a database, and analyzed with a particular focus on the presence of a patent processus vaginalis. The following items were recorded: age, gender, intraoperative findings of the processus vaginalis, e.g., unilateral vs. bilateral, patency (complete/partially obliterated), diameter of the internal inguinal ring, operative time, pain, intraoperative and postoperative complications, and recurrence rates.

\section{Surgical technique}

Basically all patients were operated under general anesthesia, and only a few had an epidural anesthesia. Local anesthesia was not used. The inguinal region was explored by an open anterior approach by or under supervision of a single surgeon (HV). Intraoperative diagnosis of a patent processus vaginalis relied on the typical anatomical features depicted in Fig. 1a-d. The cremaster muscle sheath and
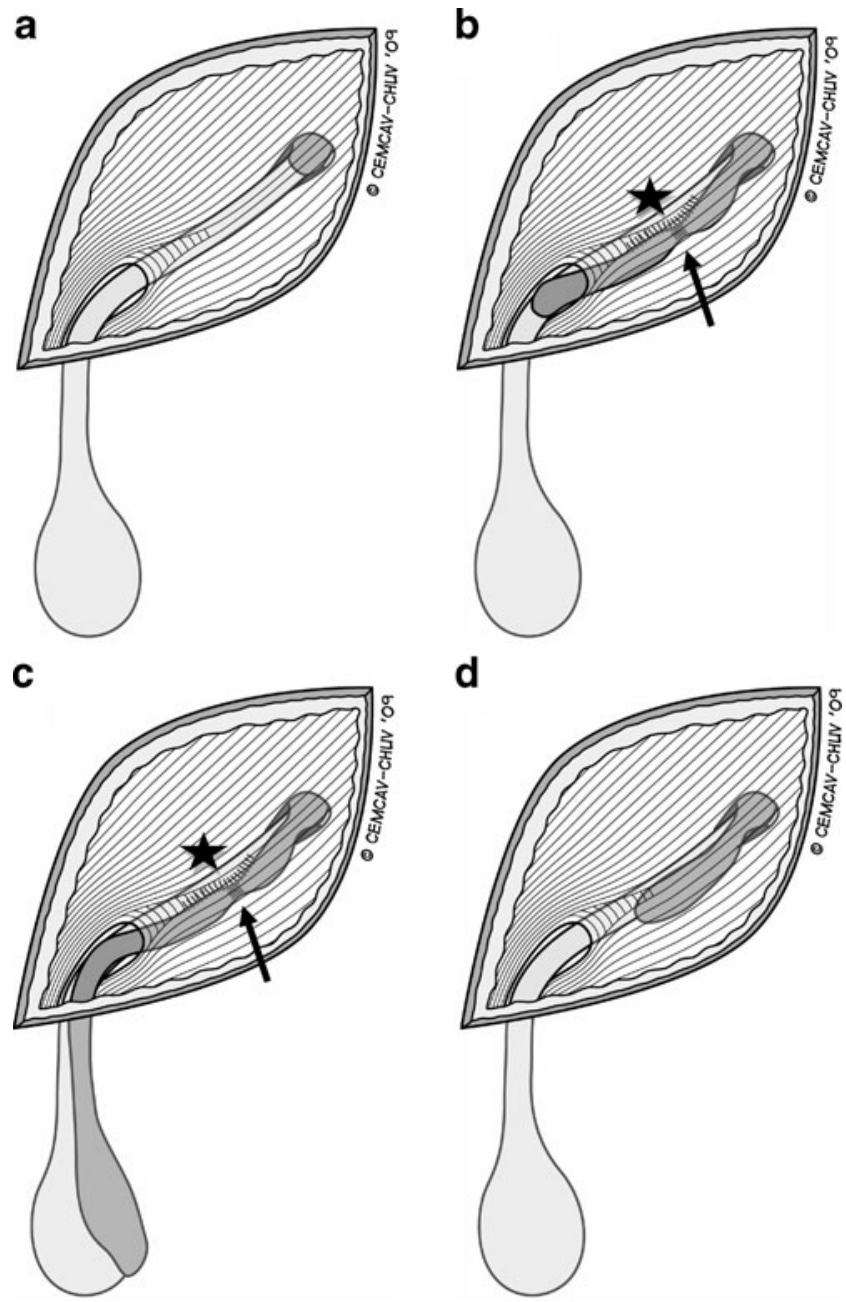

Fig. 1 Concept of patent processus vaginalis peritonei. During intrauterine development, the processus vaginalis follows the testicular descent (or round ligament) through the abdominal wall via the inguinal canal into the scrotum (or labia major). The processus vaginalis undergoes usually postnatal obliteration, except for the part covering the testes which forms the tunica vaginalis (a). Due to failed obliteration, the processus vaginalis can remain open partially (b) or completely (c). The clinical correlate is either a hydrocele or a congenital inguinal hernia. Typically, in patients with a patent processus vaginalis, the peritoneal sac is intimately fused to the spermatic cord structures (b, c; asterisk). A second characteristic feature is a circumferential fibrous rim corresponding to the proximal orifice and the usual site of postnatal obliteration (b, c; arrow). To the contrary, an acquired indirect inguinal hernia does not show any of these two characteristics and can hence be distinguished (d)

fascia were divided transversally on the anterior circumference. This allowed exposure of the peritoneal sac that is intimately fused to the spermatic cord structures. A further typical finding is a circumferential fibrous rim visualized upon opening of the hernia sac (Fig. 2a) that corresponds to the inner inguinal ring. The posterior aspect of the hernia sac was also incised allowing separation of the sac from the underlying cord structures. Finally, the hernia sac was removed after its closure at the level of the inner ring. In 

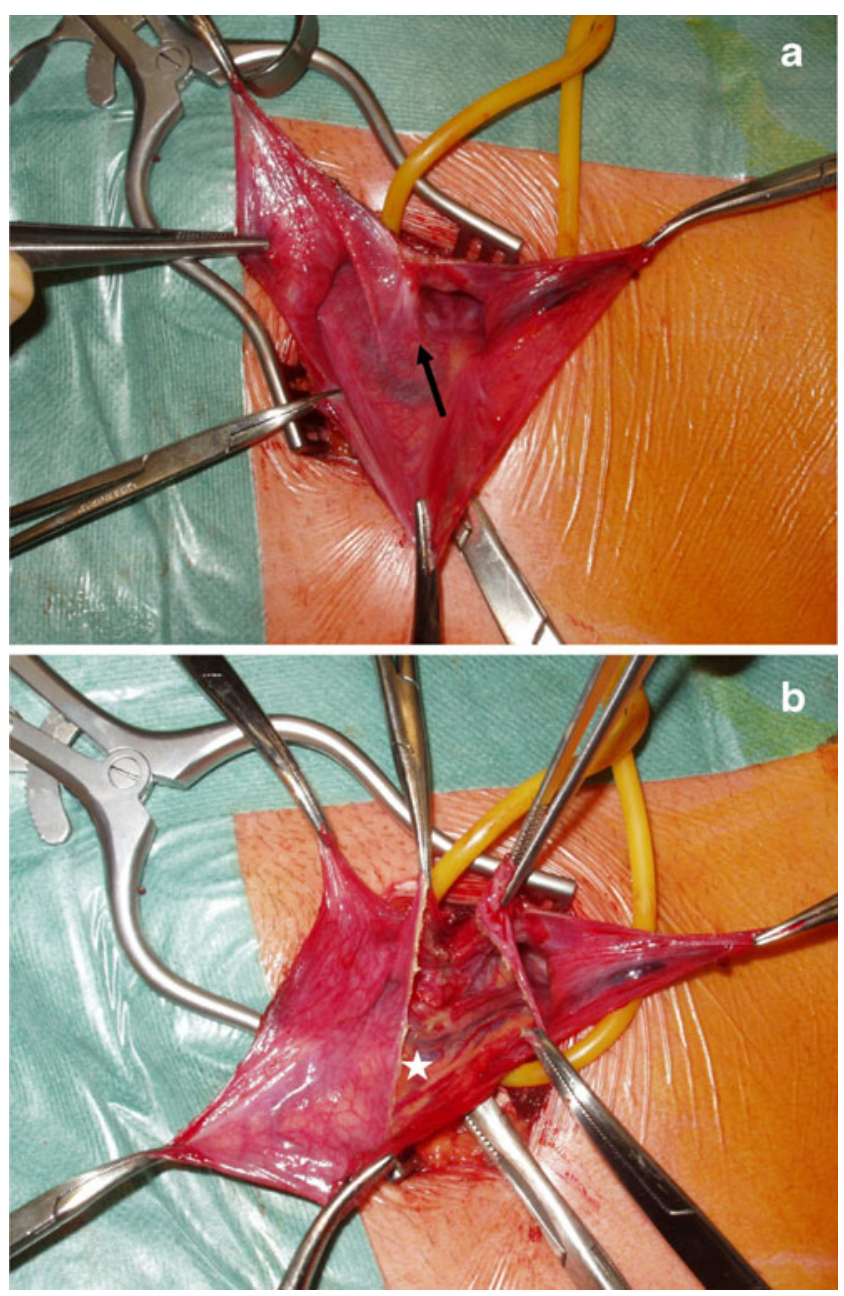

Fig. 2 Intraoperative findings of patent processus vaginalis peritonei. Intraoperative aspect of a left-sided PPV with partial obliteration. The pathognomonic circumferential fibrous rim gets visible after opening of the hernia sac (a, arrow). Incision of the posterior peritoneum allows separation of the sac from the underlying intimately fused cord structures (b, asterisk). The proximal part is transformed into an indirect sac for high ligation, while the distal part (sac remnant) is excised in toto

case of partial obliteration of the PPV (Fig. 1b), the remnant sac was usually excised in toto. If the sac extended into the scrotum (complete patency, Fig. 1c), the distal part was left open in situ. The diameter of the internal ring was always measured.

The type of reconstruction was chosen after complete dissection and formal assessment of the posterior wall. While younger patients with solid tissue and an internal ring diameter of less than $30 \mathrm{~mm}$ received a simple suture reconstruction by narrowing the inner ring, elderly patients with an enlarged internal ring and involvement of the transversalis fascia underwent tension-free mesh repair [11, 12, 16-18]. The inner ring was reconstructed by two to four interrupted stitches with non-resorbable polypropylene sutures (2.0) to a diameter of $10 \mathrm{~mm}$.
Follow-up

Regular postoperative follow-up with clinical examination was performed at 1 week, 6 months, and 12 months after surgery by the operating surgeon. The diagnosis of typical complications was based on clinical symptoms and detailed a detailed examination [19]. In addition, to document the long-term course, all patients were either clinically reassessed or at least asked to answer a standardized questionnaire in 2008. Non-responders were contacted a minimum of three times by mail and phone. Symptomatic patients were invited to come for a clinical examination.

\section{Statistical analysis}

Descriptive statistics are expressed as median and range values. Absolute numbers and percentage were used where appropriate. Continuous variables between the two groups were compared by the Mann-Whitney $U$ test. Fisher's exact test was employed for the comparison of categorical variables. A two-sided $P$ value of less than 0.05 was considered to indicate statistical significance.

We performed all analyses using SPSS 15.0 for Windows (SPSS Inc, Chicago, IL).

\section{Results}

Patients

Overall, there were 676 hernia repairs performed for 98 direct and 578 indirect hernias (Fig. 3). A patent processus

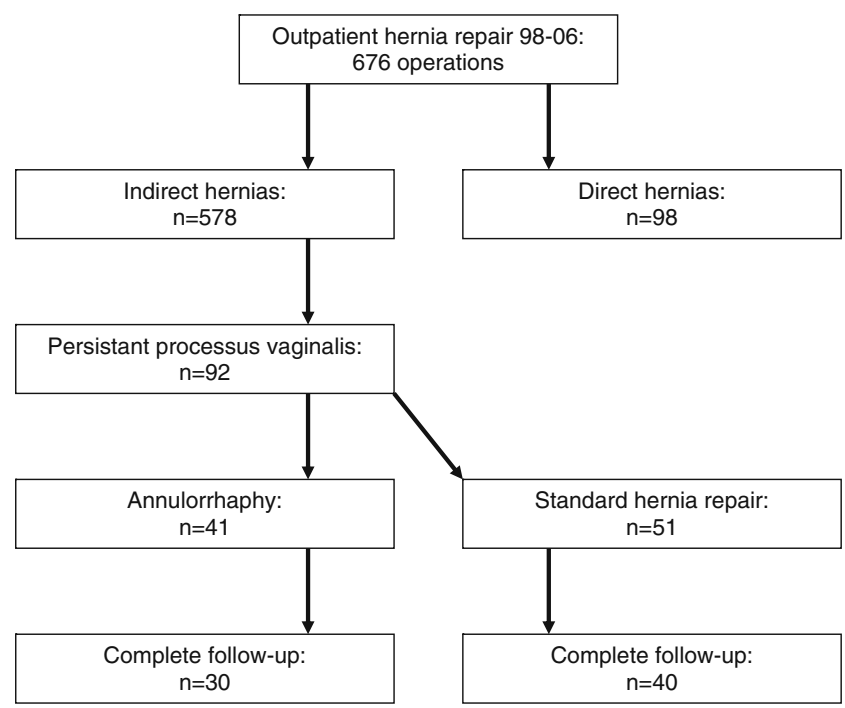

Fig. 3 Characteristics of patients undergoing ambulatory hernia repair. Characteristics of the prospective cohort of 676 consecutive patients undergoing inguinal hernia repair on an outpatient basis in a single center from January 1998 until December 2006 
Table 1 Demographics of adult patients with a patent processus vaginalis: annulorrhaphy vs. standard hernia repair

\begin{tabular}{lrrr}
\hline & Annulorrhaphy $n=41$ & Standard repair $\mathrm{n}=51$ & $\mathrm{P}$ \\
\hline Age (years) & $26(17-81)$ & $38(20-85)$ & 0.0001 \\
Right / left side & $27 / 14$ & $35 / 16$ & 0.825 \\
Patency (complete / partial) & $9 / 32$ & $22 / 29$ & 0.046 \\
Internal orifice (mm) & $22(12-43)$ & $36(15-50)$ & 0.0001 \\
Operation time (min) & $38(27-53)$ & $48(31-45)$ & 0.0001 \\
\hline
\end{tabular}

Age, diameter of the internal orifice and operation time are displayed as median (range), sidedness and patency as absolute numbers

vaginalis was found in 92 indirect hernias (prevalence $16 \%$, $92 / 578$ ) in 90 patients (one female, 89 male) with a median age of 34 years (17-85 years), whereof two patients with a bilateral patent processus vaginalis.

\section{Intraoperative findings}

A PPV was more frequently found on the right side $(67 \%, 62 /$ 92). A partial obliteration (no extension of an open processus vaginalis down to the scrotum) was detected in $66 \%$ (61/92). The internal inguinal ring was significantly larger in the 31 cases with a completely patent PPV than in the remaining 61 with a partially obliterated PPV (median diameter of $38(15-50 \mathrm{~mm})$ vs. $25 \mathrm{~mm}(12-48 \mathrm{~mm}) ; P<0.0001)$.

\section{Surgery}

According to the above-mentioned criteria 41 patients with an intact inguinal floor underwent annulorrhaphy to reconstruct a minimally enlarged inner ring. The remaining 51 patients had a standard open hernia corresponding to institutional guidelines. A tension-free mesh repair was performed in 37 patients, ten patients had a mesh-plug, and four operations were suture repairs (Shouldice). Demographics of the two comparative groups are shown in Table 1. The median operation time was significantly shorter for annulorrhaphy (38 $\mathrm{min}(27-53 \mathrm{~min})$ vs. $48 \mathrm{~min}(31-45 \mathrm{~min}), P<0.0001)$.

\section{Outcome}

In the postoperative period, we observed no testicular atrophy, wound infection, or other related complication in any patient. After a follow-up time of 1 week $(100 \%$ of patients included), 6 months (100\% of patients included), and 12 months ( $86 \%$ of patients included) one recurrence was diagnosed after annulorrhaphy and two after Lichtenstein repair. We found consistent results in a long-term follow-up after a median time of 56 months (27128 months) with no additional recurrences. Of note, only $76 \%$ of the study cohort was available for the long-term assessment and answered a standardized questionnaire. Twenty-eight percent accepted to come for an additional clinical examination. The clinical outcome is shown in Table 2. Both groups did not differ significantly with regard to recurrence, chronic pain and hypoesthesia. However, five patients in the standard repair group experienced neuropathic symptoms vs. none after annulorrhaphy $(P=0.066)$.

\section{Discussion}

This current study assessed the prevalence of a patent processus vaginalis in a large patient group undergoing ambulatory inguinal hernia repair in a single center during an 8-year period. Furthermore, the role of a tailored approach for the repair of indirect inguinal hernia caused by the presence of a patent processus vaginalis was evaluated. Based on the typical anatomical findings, a patent processus vaginalis was detected in $16 \%$ of patients with an indirect inguinal hernia, whereby two thirds of them were localized on the patient's right side. A reconstruction of the inner ring was successfully performed in patients with "small" indirect hernia, i.e., diameter of the inner ring $<30 \mathrm{~mm}$, intact transversalis fascia, young age $<30$ years.
Table 2 Outcome of adult patients with a patent processus vaginalis: annulorrhaphy vs. standard hernia repair

Follow-up (months) is expressed as median (range), all other values are absolute numbers

\begin{tabular}{lccc}
\hline & Annulorrhaphy $\mathrm{n}=41$ & Standard repair $\mathrm{n}=51$ & $\mathrm{P}$ \\
\hline Complete follow-up & $30(73 \%)$ & $40(78 \%)$ & 0.627 \\
Follow-up (months) & $56(28-113)$ & $55(27-128)$ & 0.722 \\
Recurrence & 1 & 2 & 1.000 \\
Persistent pain & 3 & 4 & 1.000 \\
An-/hypoesthesia & 5 & 9 & 0.764 \\
Neuropathic symptoms & 0 & 5 & 0.066 \\
\hline
\end{tabular}


It is estimated that up to $5 \%$ of full-term born babies have clinically relevant inguinal hernia, whereby all of them are related to an open processus vaginalis [7-10, 20-22]. With increasing age, the incidence of a patent processus vaginalis steadily falls, and approximately $10 \%$ to $30 \%$ of adults reveal an open processus vaginalis $[4,6]$. It was found that those patients are at increased risk (fourfold chance) to develop a symptomatic inguinal hernia within a 5-year follow-up compared to patients with an obliterated processus vaginalis [5]. Enlargement of the inner ring is the key process for hernia formation. If the diameter of the inner ring is larger than $30-40 \mathrm{~mm}$, it is very probable, that the posterior wall of the inguinal floor will be involved. The transversalis fascia is weakened and its defect further promotes symptomatic hernia formation. Our series demonstrates in according to the findings of the literature, that increasing age with its inherited impact of risk factors is related to larger indirect hernia.

The overall prevalence of PPV in our study cohort of symptomatic patients undergoing hernia surgery was $16 \%$. Due to its congenital formation, the processus vaginalis is firmly attached to the other cord structure which makes it very difficult to safely remove such a processus vaginalis. If left in situ, particularly in the scrotum, there is a high risk of pseudo-recurrence of the operated hernia [23]. The presence of a PPV in adults might have an important implication in endoscopic hernia repair. A complete reduction of a hernia sac by blunt dissection might be impossible due to dense adhesions down to the scrotum (Fig. 1 and 2). Furthermore, PPV can more easily be misinterpreted by endoscopic repair as the typical features of PPV (dense adhesions, fibrous rim) can hardly be seen by the posterior approach. This might partly explain why hernia "recurrence" after endoscopic hernia repair appears more frequently as an (probably persistent) indirect hernia even after previous repair of a direct hernia [23].

Tension-free mesh repair has become the standard technique for all kinds of inguinal hernia, with a wellproven low recurrence rate [24]. Nevertheless, chronic pain remains an important long-term complication of mesh implantation [12, 19, 25]. In the particular setting of a small indirect hernia due to a PPV in a young patient without any risk factors, a complete reconstruction and reinforcement of the inguinal floor may be over-treatment. Simple reconstruction of the inner ring by narrowing its diameter, a so called annulorrhaphy, might likewise apply for adults with an intact inguinal floor, and possibly reduce morbidity due to extensive dissection and mesh placement [16, 19, 25]. We implemented this concept to perform an annulorrhaphy in patients with an inguinal ring diameter of $<30 \mathrm{~mm}[11,17$, 18]. Patients with a larger diameter of the inner ring underwent standard hernia repair, mostly by performing a Lichtenstein operation. We found a significantly decreased incidence of postoperative chronic pain after annulorrhaphy. Moreover, the operative time was shorter. During long-term follow-up, recurrence rates were similar for annulorrhaphy and standard repair, respectively. Our results therefore suggest that by using a careful patient selection, annulorrhaphy may still play a role in a concept of a tailored approach for inguinal hernia repair. Selection criteria include young patient age $<30$ years, diameter of the internal ring $<30 \mathrm{~mm}$, intact inguinal floor, no peritoneal dialysis, and no risk factors, such as smoking, collagen disorders, increased intra-abdominal pressure or lifting heavy weights.

Our study is one of the rare data on the role of a PPV in adults but several limitations should be addressed. Due to high failure rates of early series of annulorrhaphy, this technique is not well reputed in adult hernia repair $[15,26]$. Long-term follow-up was only obtained in $76 \%$ of the original patient group, but short-term follow-up at 6 and 12 months was $100 \%$ and $86 \%$, respectively. This is of importance as pain and neuropathic symptoms occur typically in the short-term follow-up [19, 27-29]. However, hernia recurrence increases over time and can appear late after surgery regardless of the operation technique [30, 31]. Therefore, our true recurrence rates are likely to be somewhat higher in both comparative groups.

In conclusion, a patent PV is present in a significant number of patients undergoing inguinal hernia repair and may be an underestimated risk factor. In well-selected patients, annulorrhaphy can be considered as a functional repair with good long-term results.

Acknowledgments The authors thank Giustina Mariotti for her valuable help in data management and Philippe Clavel for drawing and providing Fig. 1a-d.

Financial support No disclosures.

\section{References}

1. Matthews RD, Neumayer L (2008) Inguinal hernia in the $21 \mathrm{st}$ century: an evidence-based review. Curr Probl Surg 45:261-312

2. Fruchaud H (1953) The effect of the upright position proper to man upon the anatomy of the inguinal region: surgical consequences; anatomic bases of surgical treatment of inguinal hernia. Mem Acad Chir (Paris) 79:652-661

3. Fruchaud H (1957) [Surgical treatment of inguinal hernias in adults.]. Bull Med 71, 293 passim.

4. Golka T, Holschneider AM, Fischer R, Blessing MH (1989) Pathogenicity of the open processus vaginalis peritonei. Z Kinderchir 44:88-90

5. van Veen RN, van Wessem KJ, Halm JA, Simons MP, Plaisier PW, Jeekel J, Lange JF (2007) Patent processus vaginalis in the adult as a risk factor for the occurrence of indirect inguinal hernia. Surg Endosc 21:202-205

6. van Wessem KJ, Simons MP, Plaisier PW, Lange JF (2003) The etiology of indirect inguinal hernias: congenital and/or acquired? Hernia 7:76-79 
7. Ein SH, Njere I, Ein A (2006) Six thousand three hundred sixtyone pediatric inguinal hernias: a 35-year review. J Pediatr Surg 41:980-986

8. Miltenburg DM, Nuchtern JG, Jaksic T, Kozinetz CA, Brandt ML (1997) Meta-analysis of the risk of metachronous hernia in infants and children. Am J Surg 174:741-744

9. Mollen KP, Kane TD (2007) Inguinal hernia: what we have learned from laparoscopic evaluation of the contralateral side. Curr Opin Pediatr 19:344-348

10. Riquelme M, Aranda A, Riquelme QM (2009) Laparoscopic pediatric inguinal hernia repair: no ligation, just resection. J Laparoendosc Adv Surg Tech A 20:77-80

11. Schumpelick V, Treutner KH, Arlt G (1994) Inguinal hernia repair in adults. Lancet 344:375-379

12. Amid PK (2005) Groin hernia repair: open techniques. World J Surg 29:1046-1051

13. Condon RE, Nyhus LM (1962) Inguinal hernia: newer trends in management. Med Sci 12:801-814

14. Nyhus LM, Condon RE, Harkins HN (1960) Clinical experiences with preperitoneal hernial repair for all types of hernia of the groin, with particular reference to the importance of transversalis fascia analogues. Am J Surg 100:234-244

15. Jess P, Hauge C, Hansen CR (1999) Long-term results of repair of the internal ring for primary inguinal hernia. Eur J Surg 165:748-750

16. Griffith CA (1984) The Marcy repair revisited. Surg Clin North Am 64:215-227

17. Miserez M et al (2007) The European hernia society groin hernia classification: simple and easy to remember. Hernia 11: $113-116$

18. Schumpelick V, Treutner KH, Arlt G (1994) Classification of inguinal hernias. Chirurg 65:877-879

19. Vuilleumier H, Hubner M, Demartines N (2009) Neuropathy after herniorrhaphy: indication for surgical treatment and outcome. World J Surg 33:841-845

20. Maddox MM, Smith DP (2008) A long-term prospective analysis of pediatric unilateral inguinal hernias: should laparoscopy or anything else influence the management of the contralateral side? J Pediatr Urol 4:141-145
21. Palmer LS, Rastinehad A (2008) Incidence and concurrent laparoscopic repair of intra-abdominal testis and contralateral patent processus vaginalis. Urology 72:297-299, discussion 299

22. Toki A, Watanabe Y, Sasaki K, Tani M, Ogura K, Wang ZQ (2003) Adopt a wait-and-see attitude for patent processus vaginalis in neonates. J Pediatr Surg 38:1371-1373

23. Lamb AD, Robson AJ, Nixon SJ (2006) Recurrence after totally extraperitoneal laparoscopic repair: implications for operative technique and surgical training. Surgeon 4:299-307

24. Collaboration EH (2000) Mesh compared with non-mesh methods of open groin hernia repair: systematic review of randomized controlled trials. Br J Surg 87:854-859

25. Aasvang E, Kehlet H (2005) Surgical management of chronic pain after inguinal hernia repair. Br J Surg 92:795-801

26. Beets GL, Oosterhuis KJ, Go PM, Baeten CG, Kootstra G (1997) Longterm followup (12-15 years) of a randomized controlled trial comparing Bassini-Stetten, Shouldice, and high ligation with narrowing of the internal ring for primary inguinal hernia repair. J Am Coll Surg 185:352-357

27. Aasvang E, Kehlet H (2005) Chronic postoperative pain: the case of inguinal herniorrhaphy. Br J Anaesth 95:69-76

28. Berndsen FH, Petersson U, Arvidsson D, Leijonmarck CE, Rudberg C, Smedberg S, Montgomery A (2007) Discomfort five years after laparoscopic and Shouldice inguinal hernia repair: a randomised trial with 867 patients. A report from the SMIL study group. Hernia 11:307-313

29. Poobalan AS, Bruce J, Smith WC, King PM, Krukowski ZH, Chambers WA (2003) A review of chronic pain after inguinal herniorrhaphy. Clin J Pain 19:48-54

30. Arvidsson D et al (2005) Randomized clinical trial comparing 5year recurrence rate after laparoscopic versus Shouldice repair of primary inguinal hernia. Br J Surg 92:1085-1091

31. Eklund AS, Montgomery AK, Rasmussen IC, Sandbue RP, Bergkvist LA, Rudberg CR (2009) Low recurrence rate after laparoscopic (TEP) and open (Lichtenstein) inguinal hernia repair: a randomized, multicenter trial with 5-year follow-up. Ann Surg 249:33-38 